\title{
Anne-Isabelle BouTon-TOUBoulic, Carlos LÉvy (éds.), Scepticisme et religion. Constantes et évolutions, de la philosophie hellénistique à la philosophie médiévale
}

Turnhout, Brepols, 2016, 300 p.

\section{Matteo Scozia}

\section{OpenEdition}

\section{Journals}

Édition électronique

URL : https://journals.openedition.org/assr/33469

DOI : $10.4000 /$ assr.33469

ISSN : $1777-5825$

Éditeur

Éditions de l'EHESS

Édition imprimée

Date de publication : 1 décembre 2017

Pagination : 289-292

ISSN : 0335-5985

Référence électronique

Matteo Scozia, « Anne-Isabelle воuтon-touвoulıc, Carlos lévy (éds.), Scepticisme et religion. Constantes et évolutions, de la philosophie hellénistique à la philosophie médiévale ", Archives de sciences sociales des religions [En ligne], 180 | octobre-décembre 2017, mis en ligne le 01 décembre 2017, consulté le 22 septembre 2021. URL : http://journals.openedition.org/assr/33469 ; DOI : https://doi.org/10.4000/ assr.33469

Ce document a été généré automatiquement le 22 septembre 2021.

(C) Archives de sciences sociales des religions 


\section{Anne-Isabelle BouTON-TOUBOULIC, Carlos LÉVY (éds.), Scepticisme et religion. Constantes et évolutions, de la philosophie hellénistique à la philosophie médiévale}

Turnhout, Brepols, 2016, 300 p.

Matteo Scozia

\section{RÉFÉRENCE}

Anne-Isabelle Bouton-TouBoulic, Carlos LÉVY (éds.), Scepticisme et religion. Constantes et évolutions, de la philosophie hellénistique à la philosophie médiévale, Turnhout, Brepols, 2016, 300 p.

1 Ce volume collectif rassemble treize études abordant le rapport entre scepticisme (courant philosophique aux implications confessionnelles et socio-politiques) et religion (païenne et chrétienne), à partir de l'Antiquité gréco-romaine jusqu'à la scolastique, en passant par l'Antiquité tardive et le haut Moyen Âge. L'ouvrage se compose de deux parties : la première se concentre sur une série de penseurs allant de Pyrrhon d'élis à Philon d'Alexandrie; la deuxième concerne saint Augustin et les retentissements de ses réflexions sur la période médiévale.

Dans la « Préface ", Anne-Isabelle Bouton-Touboulic et Carlos Levy explicitent la raison pour laquelle le rapport entre l'approche philosophique sceptique et la religion connaît un intérêt grandissant dans le débat historiographique contemporain. En effet, l'analyse de ce rapport éclaire nombre de problématiques théoriques, politiques et culturelles qui ont marqué l'Antiquité et le Moyen Âge et qui ne peuvent pas être rangées (et, par là, banalisées) sous l'étiquette de traditions ou de la « pensée sauvage », 
comme disait Lévi-Strauss. Il semble, de prime abord, qu'il y ait une distinction entre "religion populaire» et "religion des philosophes». Ce dualisme caractérise l'approche du transcendantal dès l'origine de la pensée philosophique et se répercutera sur les débats ultérieurs. Les études d'anthropologie dogmatique de Pierre Legendre, fondées sur la distinction entre pensée sauvage et discours savant, clarifient cette distinction à partir d'une perspective méthodologique qui couvre toute l'histoire de la pensée. Legendre pointe l'écart entre une approche confessionnelle du transcendant qui, ne se soucie pas du respect de principes logiques, et suit l'inclinaison de la spiritualité populaire - et une approche plus rationnelle. Plus un discours confessionnel nous éloigne du contexte empirique, plus il s'accorde avec sa propre cause. Au contraire, le discours savant n'a aucune prise sur les fidèles ou sur la communauté des croyants; il se propose d'être le soubassement logico-rationnel d'une doctrine suffisamment vraisemblable pour ce qui touche l'idée d'une entité transcendante en accord avec un récit fondateur. C'est d'ailleurs pour cette raison que les commentaires théologiques des Sentences pendant le moment scolastique n'attireront pas le fidèle ordinaire, qui subira en revanche la fascination d'un texte poétique ou des discours apocalyptiques et politiques joachimites et pseudo-joachimites ou encore de la mystique rhénane et flamande. En ce sens, la discussion entre "scepticisme et religion» est à replacer dans le cadre du rationalisme philosophique. Le but du philosophe classique ou médiéval s'intéressant à la religion est de justifier ou de discuter de façon critique le fait religieux, sans pour autant se livrer à la prédication populaire. À cet égard, les auteurs de la «Préface» soulignent «l'impossibilité de rompre tout lien avec la religion populaire, comme aussi de se confondre avec celle-ci » (p.11). Envisager le fait religieux en tant que point de départ permet de lier ces deux domaines. Mais, en même temps, l'approche rationnelle, se distinguant de celle des confessions, les sépare. L'évocation du cas de Socrate est emblématique : un excès de rationalisme (qualifié par les auteurs d'« athéisme »), qui risque de déboucher sur une subversion sociale (c'est-à-dire de saper la structure dogmatique qui encadre la norme morale), est puni de la peine capitale. Au-delà du cas de Socrate, il paraît légitime de concevoir la religion comme discours sauvage servant le contrôle social. Un autre élément qui permet de mieux cerner le périmètre de la discussion provient de la réflexion autour d'Héraclite et de son scepticisme face à la ritualité religieuse : une approche critique qui reviendra sur le devant de la scène lorsqu'il sera question, dans le stoïcisme impérial, du recours à l'allégorie pour expliquer le transcendant et qui influencera considérablement la vision sceptique du fait religieux.

Bien que le scepticisme soit considéré, à juste titre, comme courant de pensée dont les éléments se retrouvent dans des époques données, l'approche savante du discours religieux demeure la toile de fond du courant sceptique. Ce dernier est présent dans le contexte chrétien sous différentes formes, ce qui confirme sa particularité. Le fidéisme de Philon d'Alexandrie englobe dans sa proposition le dualisme entre scepticisme et religion, minorant le discours savant, réputé inférieur à la foi et au saut spirituel que celle-ci rend possible. La foi peut être, elle aussi, rationnelle, dans la mesure où elle offre des catégories logiques alternatives à celles du discours empirique. Le transcendantal est à même de s'imposer lorsqu'il «rabaiss[e] les prétentions de la raison humaine devant la grandeur du créateur, qui est au-delà de toute connaissance " (p. 21). Ce sont là les fondements de la Christianitas, ce grand projet politique et culturel, postulant la coïncidence de la doctrine chrétienne avec la normativité sociale, qui deviendra le manifeste culturel de l'aire géographique qui aujourd'hui inclut l'Europe, 
une partie de l'Afrique et du Proche Orient. Des auteurs tels qu'Eusèbe de Césarée et Augustin d'Hippone œuvrent au redimensionnement de ce discours rationnel sur le religieux dans le nouveau contexte chrétien (p. 22-26). Une voie intermédiaire entre approche sceptique classique et exigences culturelles et sociales chrétiennes émergera chez certains auteurs du Moyen Âge tardif qui, toujours à l'intérieur d'un discours savant, essayeront de combler le fossé entre le raisonnement sceptique et la foi religieuse.

4 Anna Maria Ioppolo, de son côté, passe au crible la stratégie argumentative de Carnéade contre la prétention stoïque de considérer la divination en tant que technè. Pour les stoïques, la divination ne devrait concerner que les événements fortuits, pour la prévision desquels l'analyse scientifique n'est pas opératoire. La critique de Carnéade vise les causes : la prévision de tout événement dépend de la connaissance des causes éternelles. Or, l'existence de ces dernières est réfutée. Cette approche sceptique s'applique aussi à un être divin, qui ne serait à même de connaître aucun événement futur pour la simple raison que les conditions d'une telle prévision, à savoir les causes éternelles, n'existent pas.

Du scepticisme pur de Carnéade l'on passe au fidéisme de Philon d'Alexandrie. Carlos Lévy met en lumière la spécificité de cette approche, qui ne saurait être réduite ni au scepticisme de Sextus Empiricus ni à l'idée de monothéisme unique avancée par le christianisme (Lévy parle de "catholicisme»). Philon propose une approche plus mitigée : le scepticisme est un déséquilibre entre la puissance absolue de Dieu et les limites humaines dans la connaissance. L'approche religieuse se réduit dès lors à une sorte de théologie négative, selon laquelle la raison humaine n'est pas en mesure de comprendre l'être divin.

6 Le platonisme de Plutarque, objet de la contribution de Mauro Bonazzi, n'est pas apparenté au fidéisme de Philon, car la fracture entre raison et foi que l'on retrouve chez le premier est absente de la réflexion du second. Selon Bonazzi, Plutarque est un tenant d'un "scepticisme métaphysique ». Ce scepticisme se résume à l'attitude qui consiste à interroger sans cesse les réalités sensibles afin de repérer les caractéristiques que Platon attribuait aux Intelligibles et que Plutarque associe au divin. Si Dieu est l'Être suprême, il n'est pas connaissable en tant que tel: il est des vérités qui ne peuvent pas être réfutées, bien qu'elles soient inconnaissables. L'objectif est moins la vérité en soi que l'incapacité structurelle de la raison humaine de connaître des réalités dépassant certaines limites. Cette espèce de dogmatisme est critiqué par Sextus Empiricus. Au lieu de justifier rationnellement l'existence de quelque chose que l'on ne peut pas prouver, il serait plus utile de s'appuyer sur une «théologie passive et nondogmatique », qui accepte comme prémisse apriori l'existence de Dieu, la vénération qui en suit et la conviction d'être impliqué dans les affaires mondaines.

7 Emidio Spinelli et Stéphane Marchand révèlent la portée sociale de la pensée de Sextus Empiricus. La piété populaire peut être appréhendée comme une question séparée de celle de l'existence de Dieu. En employant des catégories contemporaines, nous pourrions soutenir dans cette perspective que le discours de fondation - et la théologie politique qu'il implique - est autonome par rapport aux obligations morales qu'il peut impliquer.

8 Comme le rappelle Brigitte Pérez-Jean, autant le scepticisme que la théologie négative font appel à la «notion d'ineffable, d'indicible » (p. 119) pour justifier l'impossibilité de connaître une réalité transcendantale. Cette impossibilité est exprimée 
linguistiquement et conceptuellement par le biais de l'« a-privatif ». Il en résulte que le scepticisme et la théologie négative sont les deux faces d'une même pièce: des approches rationnelles, respectivement, de l'époque classique et de celle chrétienne. L'essence du courant sceptique semble être mise en lumière par Lactance dans De opificio Dei et dans Divinae Institutiones. L'étude de Gábo Kendeffy met à cet égard l'accent sur le fait que, pour Lactance, le scepticisme ne conduit à - et n'affirme aucune vérité, hormis celle de montrer les différences et les limites des doctrines philosophiques qui cherchent en vain à aborder le transcendant. Par contre, la doctrine chrétienne - «la vraie philosophie [...] au sens où elle est le vrai par rapport à la philosophie qui est un faux-semblant» (p. 154) - affirme l'existence d'une vérité qui, tout en se révélant dans sa plénitude existentielle, ne peut être que partiellement saisie par l'esprit humain, sans que cela la rende moins vraie ou moins divine. La contribution de Jesús Hernández Lobato, centrée sur Grégoire de Nysse, porte, elle aussi, sur l'affirmation de cette vérité, en mitigeant le dualisme entre scepticisme et religion. À l'instar de Brigitte Pérez-Jean, l'auteur s'intéresse à la théologie négative, conçue comme expression chrétienne du scepticisme épistémologique.

La synthèse de la pensée de l'Antiquité et de l'Antiquité tardive est due à Augustin d'Hippone qui s'impose comme un véritable filtre pour l'époque médiévale suivante. Les contributions d'Anne-Isabelle Bouton-Touboulic, Isabelle Bochet et Giovanni Catapano en éclairent les aspects fondamentaux : premièrement, l'inconciliabilité entre scepticisme et religion, dans la mesure où le premier est un "dogmatisme négatif " (avec une référence exclusive aux Académiques et à leur porte-parole, Cicéron); deuxièmement, la conviction d'une incompatibilité entre approche sceptique et foi chrétienne ; troisièmement, la démonstration logique, et non seulement doctrinale, d'un tel refus.

Les dernières contributions portent respectivement sur Jean de Salisbury et Jean Gerson et nous mènent à l'époque médiévale, en décrivant la façon selon laquelle ceuxci s'insèrent dans un débat revêtant une grande valeur aux yeux des penseurs médiévaux : le rapport entre la préscience divine et la liberté humaine. À première vue, il semblerait que le probabilisme évoqué par Jean de Salisbury lorsqu'il est question de la préscience soit le fruit de l'influence sceptique, ce qui l'obligerait à réfuter la proposition augustinienne. Le commentateur, Christophe Grellard, insiste sur une vision morale pour laquelle une reconnaissance des limites dans la connaissance représente une forme d'humilité masquée par une approche théorétique. Quant à Jean Gerson, comme le souligne Alice Lamy, il prône une "certitude probable ", et ce, en raison des limites consubstantielles à l'homme, qui ne lui permettent pas d'affirmer, de façon absolument certaine, quoi que ce soit, même pas dans le domaine de la morale, contrairement à ce que soutiendra, deux siècles plus tard Anthony Terill, héritier de Gerson dans ce même domaine.

11 Les contributions de cet ouvrage bien structuré abordent les aspects fondamentaux de la question dans la période examinée. Les références ponctuelles aux sources primaires et secondaires, rassemblées dans une riche bibliographie finale, permettent au lecteur de se pénétrer des propositions des auteurs analysés. De plus, l'excellente préface, non seulement restitue l'unité de l'ensemble, mais en projette les contenus vers une discussion plus vaste s'étendant à l'époque moderne et contemporaine, en stimulant ainsi la réflexion pour d'ultérieures recherches. 